\title{
Váriance component analysis of skin and weight data for sheep subjected to rapid inbreeding
}

\author{
Frank H. Shaw ${ }^{a *}$, J.A. Woolliams ${ }^{b}$ \\ a Department of Ecology, Evolution and Behavior, University of Minnesota, 1987 \\ Upper Buford Circle, St Paul, MN 55108, USA \\ b Roslin Institute (Edinburgh), Roslin, Midlothian EH25 9PS, UK
}

(Received 5 November 1997; accepted 27 November 1998)

\begin{abstract}
A variance component analysis was carried out on data from a 20year experiment in the rapid inbreeding of purebred and crossbred lines of three hill breeds of sheep. Parent offspring matings were made over several generations to produce inbreeding coefficients in lambs of up to 0.59. The traits chosen for analysis were the live weights at 24 and 78 weeks of age and the ratio of the densities of secondary and primary skin follicles. A complete model of intralocus allelic effects was carried out with both additive genetic variance and dominance variance. The latter was partitioned into components arising from loci which were homozygous by descent and those that were not. Inbreeding depression was fitted as a covariate. This model has not been attempted previously in livestock populations. Crossbred animals were found to exhibit more dominance variance than purebred animals. Though partitioning of the dominance variance was possible in some of the data sets considered, estimation of the novel quadratic components was difficult and provided little evidence of homozygous dominance variance as distinguished from the familiar random dominance variance (that arising in randomly mated populations). A pooled dominance model is proposed in which inbred dominance effects have the same variance as random dominance effects. For live weight the results suggested that the genetic architecture involved many loci with deleterious recessive alleles, but for the ratio of follicle density there was no clear explanation for the results observed. (c) Inra/Elsevier, Paris
\end{abstract}

inbreeding depression / dominance variance / restricted maximum likelihood / variance components / sheep

* Correspondence and reprints

E-mail: fshaw@evolution.umn.edu 
Résumé - Analyse des composantes de variance pour des données de poids et de peau concernant des moutons soumis à une consanguinité rapide. Une analyse des composantes de variance a été effectuée sur des données provenant d'une expérimentation de 20 ans sur la consanguinité rapide de lignées pures et croisées issues de trois races ovines de montagne. Des accouplements entre parents et descendants ont été effectués sur plusieurs générations en vue de produire des coefficients de consanguinité élevés (jusqu'à 0,59 ) chez les agneaux. Les caractères choisis pour l'analyse ont été les poids vifs à 24 et 78 semaines d'âge et le rapport entre les densités de follicules cutanés secondaires et primaires. Un modèle complet des effets alléliques intralocus a été établi avec à la fois une variance génétique additive et une variance de dominance. Cette dernière a été partitionnée en composantes provenant de loci homozygotes par descendance mendélienne ou non. La dépression de consanguinité a été considérée en covariable. Ce modèle n'a pas été tenté précédemment sur les populations d'animaux domestiques. Les animaux croisés ont manifesté plus de variance de dominance que les animaux purs. Bien que la partition de la variance de dominance ait été possible dans quelques-uns des fichiers considérés, l'estimation des nouvelles composantes quadratiques a été difficile et n'a pas fourni de preuve flagrante que la variance de dominance chez les homozygotes doive être distinguée de la variance de dominance classique. Un modèle de dominance regroupée est proposé dans lequel les effets de dominance chez les consanguins ont la même variance que sur l'ensemble de la population. En ce qui concerne le poids vif, les résultats suggèrent que l'architecture génétique implique de nombreux loci avec des allèles récessifs délétères mais que cela ne semblait pas être le cas pour le rapport des densités de follicules. (c) Inra/Elsevier, Paris

dépression de consanguinité / variance de dominance / maximum de vraisemblance restreint / composantes de variance / mouton

\section{INTRODUCTION}

The genetic analysis of populations undergoing rapid inbreeding is of interest because the opportunity for protective mutations or haplotypes to accumulate and obscure our view of the genetic mechanisms involved is minimized. The principal phenomena predicted from inbreeding are the reduction of genetic variation within families and the disappearance of heterozygosity. When associated with dominant gene action this results in inbreeding depression. Inbreeding depression and its seeming inverse, the heterosis obtained through crossing of lines, have received much attention over the whole of this century [10,31].

Nevertheless, the interpretation of these phenomena in terms of genetic variances and covariances has remained a thorny problem. Harris [9] and Cockerham [3] developed complete mathematical models for the genetic variance of non-random mating populations. These models were used to predict gene frequency changes in populations undergoing selection [4], and, while some attempts were made to apply them to agricultural populations [5], the models have for the most part remained computationally too intensive or the testing of them empirically too demanding to be of practical use. In this paper we report the results of a complete variance component analysis of an experiment carried out between 1958 and 1974 in Scotland. Thorough analysis of inbreeding depression and heterosis was possible previously, and these have been reported for fleece and skin data $[29,30]$, weight [25], and measures of body size, reproduction, fertility and profitability [26-28]. However, a variance component analysis including dominance was not possible before now. 


\section{MATERIALS AND METHODS}

\subsection{Design and measurements}

Details of the breeding designs and the methods employed in this experiment are given by Wiener [24] and Woolliams and Wiener [30]. In brief, the breeding scheme was as follows: six rams and approximately 72 ewes of each of three hill breeds (Scottish Blackface, South Country Cheviot and Welsh Mountain) were obtained from a variety of different flocks in 1955. These were used as the foundation animals in the pedigrees. All nine possible purebred and reciprocally crossbred matings were made. These were denoted $F_{1}$ for crossbred and $O_{1}$ for purebred matings. These mating combinations (e.g. Blackface $\times$ Cheviot) are referred to as groups. Subsequently, within each group, $F_{1}$ or $O_{1}$ females were mated to unrelated males, producing $\mathrm{F}_{2}$ and $\mathrm{O}_{2}$ offspring. Inbred crosses were then carried out within the nine groups between offspring and younger parent to produce as many as 27 lines per group. The pattern of offspring with younger parent matings was carried on whenever possible for 10 years, resulting in coefficients of inbreeding of dams as high as 0.375 and coefficients of inbreeding of lambs as high as 0.59 . Finally, the separate lines that remained were crossed within the purebred and crossbred groups. A subpedigree consisting of nine lines from the purebred Blackface group is presented in figure 1 .

In this study, we examine three traits: the fleece trait $N_{s} / N_{p}$, the ratio of the secondary follicle density to the primary follicle density, and weights at 24 and 78 weeks. Of the many traits measured during this experiment, the $N_{s} / N_{p}$ trait was chosen because of the nearly linear relationship previously observed between its mean and inbreeding coefficient. In contrast, the weight traits tended to show less inbreeding depression for high levels of inbreeding than for moderate levels. The weight traits were chosen because of the large number of lambs measured for them (730 purebreds in three groups and 1480 crossbreds in three groups). The $N_{s} / N_{p}$ measurements were made on all lambs for the $F_{2} / \mathrm{O}_{2}$ generation onwards until the third inbred generation $(F=0.5)$ using estimation techniques described by Carter and Clarke [2]. The weight data were analyzed for female lambs only, but these data were run for the full length of the experiment and included lambs with the highest inbreeding level $(F=0.59)$ as well as the line cross lambs.

\subsection{Statistical model and method}

The' mixed linear model,

$$
y=X \beta+a_{i}+a_{j}+d_{i j}+e
$$

is made up of fixed effects, $\beta$, and random effects including additive allelic effects $a_{i}$, a dominance effect for the interaction between the alleles $i$ and $j$, $d_{i j}$, and a residual effect $e$. If Hardy-Weinberg frequencies hold, we have the following constraints:

$$
\mathrm{E}\left(a_{i}\right)=\mathrm{E}\left(d_{i j}\right)=0
$$


where the expectation is taken over all alleles segregating at a single locus. From these it follows that $\mathrm{E}\left(a_{i} d_{i j}\right)=0$. We can therefore write $\mathrm{E}(y)=X \beta$ and

$$
\operatorname{var}(y)=2 \mathrm{E}\left(a_{i}^{2}\right)+\mathrm{E}\left(d_{i j}^{2}\right)
$$

where the first term on the right-hand side is commonly denoted $V_{A}$ and the last $V_{d}$. If $y$ represents a vector of related but not inbred individuals in a population, we can write the covariance between any two individuals in $y$ as a linear combination of these two variance components where the coefficients are based on probabilities that the individuals share alleles or combinations of alleles at a given locus [8].

If the vector $y$ contains individuals that are inbred, i.e. individuals with nonzero probabilities of carrying two identical alleles descended from a common ancestor at a given locus, the situation becomes more complicated. We must now account for the non-vanishing presence of the term $d_{i i}$ in our equations since homozygotes increase at the expense of heterozygotes. Thus, for an individual randomly chosen with an inbreeding coefficient of $F$ with respect to the base population,

$$
\mathrm{E}\left(d_{i j}\right)=F \times \mathrm{E}\left(d_{i i}\right)
$$

where $F$ is the inbreeding coefficient, and

$$
\mathrm{E}(y)=X \beta+\mu_{F}=X \beta+F \times \mathrm{E}\left(d_{i i}\right)
$$

The variance of $y$ must now be partitioned into three more components of variance beyond those already mentioned $[9,23]$. These include the complete homozygous dominance variance,

$$
D_{2}^{*}=\mathrm{E}\left(d_{i i}^{2}\right)-\left(\mathrm{E}\left(d_{i i}\right)\right)^{2}
$$

and the expectation of the squared inbreeding depression effects,

$$
H^{*}=\left(\mathrm{E}\left(d_{i i}\right)\right)^{2}
$$

The covariance between additive effects and their associated homozygous dominance effects is non-zero $\left(\mathrm{E}\left(a_{i} d_{i i}\right) \neq 0\right)$ and upon inbreeding there is a need to account for this covariance

$$
D_{1}=\mathrm{E}\left(a_{i} d_{i i}\right)
$$

Again, the expectations involving homozygotes are taken over all alleles segregating at a single locus using the distribution of alleles in the base generation. The terminology used here is taken from Cockerham and Weir [4]. To emphasize the difference between homozygous dominance effects and dominance effects in the context of random mating with no inbreeding, we name variance of the latter $V_{d r}$ or random dominance variance [7]. Assuming no epistasis, we use these same symbols $\left(V_{A}, V_{d r}, D_{1}, D_{2}^{*}, H^{*}\right.$ ) in what follows to designate the sum of the per locus variances and covariances (given earlier) over an arbitrary number of loci. In the case of the squared inbreeding depression effects, we have

$$
H^{*}=\sum^{\text {loci }}\left(\mathrm{E}\left(d_{i i}\right)\right)^{2}
$$


the sum of the squared per locus inbreeding depressions. If the per locus inbreeding depressions are all of similar small values and there are very many loci, $H^{*}$ can be vanishingly small even when the inbreeding depression is large. It is also noted that if the trait is controlled by a single locus, $H^{*}$ will be the square of the inbreeding depression as calculated by regression of the phenotype on the inbreeding coefficient.

The variances of and covariances between individuals of known pedigree are expressed as linear combinations of these five variance components with coefficients based on the appropriate probabilities of identity of alleles by descent. The probability measures involve combinations of four alleles in two individuals [3] of which there are 16 if maternal and paternal gametes are distinguished and nine if they are not. In the case of the present analyses, loci affecting the trait are assumed to be autosomal, so the nine probability measures are sufficient. Cockerham [3] and Smith and Maki-Tanila [20] gave elegant recursive algorithms for finding these probabilities and in the latter case, finding directly the inverse of the covariance matrix of an expanded list of allelic additive and dominance effects. Here, we used Cockerham's approach to write the matrix $V$ or phenotypic covariance matrix,

$$
V=\operatorname{var}(y)=V_{A} \mathbf{A}+V_{d r} \mathbf{D}+D_{1} \mathbf{M}_{\mathbf{D}_{1}}+D_{2}^{*} \mathbf{M}_{\mathbf{D}_{2}^{*}}+H^{*} \mathbf{M}_{\mathbf{H}^{*}}+V_{e} \mathbf{I}
$$

where the matrices $\mathbf{A}, \mathbf{D}, \mathbf{M}_{\mathbf{D}_{1}}, \mathbf{M}_{\mathbf{D}_{2}^{*}}$ and $\mathbf{M}_{\mathbf{H}^{*}}$ are the appropriate relationship matrices. In the case of the weight data, a maternal environmental variance component and an appropriate incidence matrix were also added.

The restricted log likelihood function,

$$
l_{1}=-\frac{1}{2}\left[\text { const }+\log |\mathbf{V}|+\log \left|\mathbf{X}^{\prime} \mathbf{V}^{-1} \mathbf{X}\right|+(y-\mathbf{X} \beta)^{\prime} \mathbf{V}^{-1}(y-\mathbf{X} \beta)\right]
$$

where $\beta$ is the generalized least squares solution for the fixed effects, was maximized in the components of variance using the Fisher scoring algorithm $[14,18]$. The regression of the phenotype on the inbreeding coefficient $F$ is also included as a covariate which, in the absence of selection bias, will predict the inbreeding depression.

In the Fisher scoring algorithm, the inversion of $\mathbf{V}$ cannot be avoided and this restricts its use to relatively small or felicitously structured data sets such as those here. Variance component analysis, using the approach of Smith and Maki-Tanila [20] for the inversion of the mixed model equation coefficient matrix $\mathbf{C}$ along with recently developed likelihood maximization algorithms $[15,16]$, is plausible for larger data sets, although the dimension of $\mathbf{C}$ might become very large [20].

All of the data were analyzed with year of birth included both as a random and a fixed effect. The results of these analyses were not qualitatively different, with significant year variation but no long-term trend. Reported results in all cases are from analyses in which year of birth was included as a fixed effect.

Separate variance component analyses were run on the six different purebred and crossbred combinations. To detect more general behavior and to boost sample sizes, the three crossbred combinations were combined into one crossbred data set and the three purebreds were combined into a purebred data set. In 
these combined analyses, a different covariate was fitted for inbreeding depression on each purebred and on each crossbred combination. Different levels for the other fixed effects were also included so that the only constraint present in the combined analyses that was absent from the separate analyses was that all groups were assumed to have the same genetic, maternal environmental and residual variances. Likelihood ratio tests were used to evaluate the significance of this constraint. Whenever fixed effect estimates (e.g. inbreeding depression estimates) from different analyses were compared, the analyses were assumed to be independent.

As well as pooling the purebreds and the crossbreds, the potential power of the analysis was also increased by combining $V_{d r}$ and $D_{2}^{*}$ into an agregate dominance component, $V_{D}$, associated with the combined relationship matrix $\mathbf{D}+\mathbf{M}_{\mathbf{D}_{2}^{*}}$. Since in this model the dominance variance is not partitioned into separate homozygous and random components, we call it the 'pooled dominance' model. It includes, along with $V_{A}$ and $V_{D}$, the covariance between additive and homozygous dominance effects $D_{1}$.

In all analyses, significance levels for components were tested by a likelihood ratio test in the following order: $V_{e}, V_{A}, V_{m}$ (for weight), $V_{d r}, D_{2}^{*}$ and $D_{1}$. Standard errors increased as more components were added to the model. The standard errors reported are those corresponding to when all components are fitted, so that they do not reflect the levels of significance attributed by the likelihood ratio test that was used to test for a particular component's presence. Likelihood ratios were compared to the appropriate $\chi^{2}$ distributions, i.e. a 50:50 mixture of $\chi^{2}(0)$ and $\chi^{2}(1)$ for null hypotheses of a single variance component on the boundary of the parameter space and $\chi^{2}(p)$ for $p$ variance components constrained in the interior [19].

The decision to analyze separately purebred and crossbred data was made because a combined analysis would constrain the variance components from very different populations to be the same. The constraint that this would be the case in the combined purebred data alone was found to be highly significant (see Results). An analysis including all animals would be feasible computationally, though difficult.

Recently, several studies have addressed the problem of analyzing crossbred data $[11,12,22]$. The methods which have been developed use the variance components associated with the constituent purebred parental populations and, in the case of dominance, variance components associated with the crossbreds, to predict genetic values [11]. Estimation of the 26 covariance components associated with a general two breed crossbreed pedigree has not yet been attempted; however, the theory is fully developed and methods such as those employed here would suffice. In this paper, however, we do not include purebred and crossbred genotypes in the same data set and thus have no need to calculate purebred by crossbred genotypic covariances. Crossbred groups sharing a purebred parental origin are included in a single analysis, but the covariances between individuals in different groups would be very small since no mating takes place between the groups in the many generations after they are established. We therefore assume the groups to be independent and take the $F_{2} / O_{2}$ generation to represent the base population for each group. In so doing, we reduce the number of covariance components for two purebred groups and its associated crossbred group from 26 to 15 . 


\subsection{Simulation study}

Although strict attention was paid that no artificial selection should take place during the experiment, it was unavoidable that as the levels of inbreeding increased, many of the individual lines died out (figure 1). This natural selection clearly favors lines that exhibit less inbreeding depression for fitness traits. A simulation study was carried out to assess the affect on variance component estimation of loss of lines due to natural selection.

Populations of potentially 500 were simulated based on ten seven-generation pedigrees similar to those found in the experiment and shown in figure 1. Each pedigree consisted of eight unrelated founders mated in two groups of one sire and three dams. The second generation consisted of six pairs of full-sibs in two half-sib groups. The half-sib groups were then crossed to produce six noninbred progeny in the third generation. These third-generation individuals were crossed with one of their parents to produce the first inbred generation (fourth generation). This crossing was followed by three more generations of offspring by youngest parent mating. After the first generation, then, each individual was associated with, and crucial to the continued propagation of one of six different lines.

Each founder was assigned two unique alleles at each of 30 loci (480 independent alleles in each of ten pedigrees per replicate data set). Since the alleles assigned to each founder were unique, homozygosity at a locus could only occur when the alleles were identical by descent. Under the full genetic model, correlated values for additive $\left(a_{i}\right)$ and homozygous dominance $\left(d_{i i}\right)$ effects were sampled for each allele from

$$
\left(\begin{array}{c}
a_{i} \\
d_{i i}
\end{array}\right) \sim N\left(\begin{array}{ccc}
0 & \frac{V_{A}}{2 n l o c} & \frac{D_{1}}{n l o c} \\
\frac{D_{1}}{n l o c} & \frac{D_{2}^{*}}{n l o c} & \frac{1}{n l o c}
\end{array}\right)
$$

where $n l o c=30$ is the number of loci, and id $=-0.5$ is the inbreeding depression. For these simulations, $V_{A}=0.2$ and $D_{2}^{*}=0.5$. Each non-identical combination of alleles within a locus was given a random dominance effect $\left(d_{i j}\right.$ for alleles $i$ and $j$ ) drawn from a normal distribution with mean zero and variance $V_{d r}=0.2$. Transmission of alleles at each locus from one generation to the next was simulated by Mendelian segregation and free recombination into gametes. Phenotypes were calculated as the sum of the genetic values from a combined pair of gametes (the genotypic value) to which was added an independent environmental effect with mean zero and variance $V_{E}=0.3$.

Beginning in the second generation, individuals (and consequently the lines derived from them) were culled based on a linear function of phenotypic value. Four such selection schemes were simulated. In the first scheme (I), no selection was imposed. In the second scheme (II), the lowest trait value in a given generation was culled with probability 0.15 , the highest trait value with probability 0.125 , and the intermediate trait values with probability based on a linear combination of these two. Schemes III and IV were similar with, respectively, 0.2 and 0.25 probability of culling of the lowest trait value for each generation, and zero probability of culling for the highest trait value. 


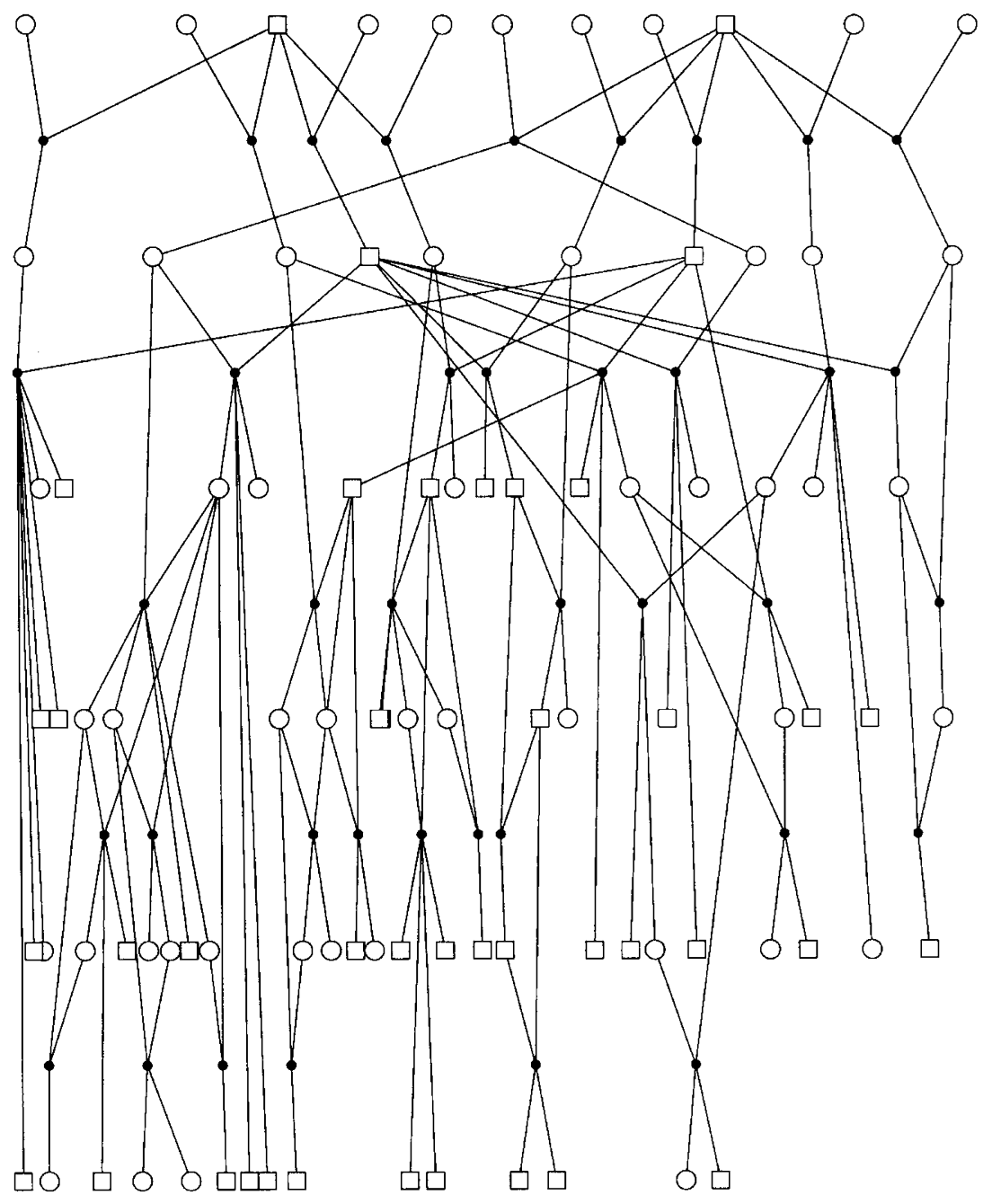

Figure 1. Pedigree for one of three independent sets of lines for the Scottish Blackface breed. This is the pedigree used for analysis of $N_{s} / N_{p}$ data which omits line cross lambs. Rams are represented as squares and ewes are represented as circles. When more than two progeny appear from the same mating, the progeny were produced in different years. Matings which produced no measurable progeny are not shown. Only two of the possible eight inbred lines reached the maximum level of inbreeding. 


\section{RESULTS}

\subsection{Simulations}

The results of the simulation study are presented in table $I$. The inbreeding depression estimate is biased upwards as selection becomes more intense and more lines with lower mean phenotypic values are lost. The variance components appear to be little affected except in that the standard deviations on the mean estimates grow with data sets reflecting higher levels of selection. These data sets are not only smaller but they lack information on animals at high levels of inbreeding.

Table I. Effect of selection on variance component and inbreeding depression estimates observed in simulation study.

\begin{tabular}{lccccccc}
\hline $\begin{array}{l}\text { Selection } \\
\text { scheme* }^{*}\end{array}$ & $\begin{array}{c}\text { Mean number } \\
\text { of records }\end{array}$ & $V_{a}$ & $V_{d r}$ & $D_{1}$ & $D_{2}^{*}$ & $H^{*}$ & $\begin{array}{c}\text { Inbreeding } \\
\text { depression }\end{array}$ \\
\hline Initial & 50.00 & 0.2 & 0.2 & -0.1 & 0.5 & 0.008 & -0.5 \\
I & 50.00 & 0.198 & 0.186 & -0.091 & 0.493 & -0.078 & -0.501 \\
SE & & $(0.002)$ & $(0.005)$ & $(0.005)$ & $(0.022)$ & $(0.031)$ & $(0.005)$ \\
II & 38.49 & 0.190 & 0.208 & -0.086 & 0.474 & -0.045 & -0.470 \\
SE & & $(0.002)$ & $(0.006)$ & $(0.006)$ & $(0.024)$ & $(0.033)$ & $(0.007)$ \\
III & 37.08 & 0.198 & 0.202 & -0.096 & 0.506 & -0.030 & -0.433 \\
SE & & $(0.002)$ & $(0.006)$ & $(0.006)$ & $(0.030)$ & $(0.036)$ & $(0.008)$ \\
IV & 34.52 & 0.197 & 0.209 & -0.096 & 0.455 & 0.076 & -0.411 \\
SE & & $(0.002)$ & $(0.007)$ & $(0.007)$ & $(0.030)$ & $(0.041)$ & $(0.009)$ \\
\hline
\end{tabular}

Estimates of genetic parameters for four selection regimes of increasing intensity. Data were generated on ten fourth-generation pedigrees of size 50 similar to those found in the experiment and pictured in figure 1. Results are for 500 replicate data sets.

${ }^{*}$ Details are given in the Methods section; ${ }^{* *}$ the number of records observed declines with increasing selection. SE: standard error.

\subsection{Fixed effects for $N_{s} / N_{p}$}

In earlier analyses, when all observations were included in a single data set, the fixed effects found to be affecting this trait were dam and lamb inbreeding depression [29] and year of birth. The same fixed effects were fitted in this analysis. The estimates for inbreeding tend in the expected directions (decline in value with additional inbreeding of the dam and the lamb). The estimate for inbreeding depression of the lamb (table II) for each of the six groups was rarely more than a single standard deviation from zero, probably due to the small sample sizes of the single group data sets (purebreds had 145 to 189 observations per group; crossbreds had 300 to 361 per group) and to the lack of high levels of inbreeding either among the observed lambs or among their mothers. However, the consistent negative value supports the previous analysis and, pooled over groups, the decline is $-0.52 \pm 0.20$. The effect of the inbreeding of the dam 
(results not shown) was less pronounced. Pooled over groups, dam inbreeding of $25 \%$ resulted in a trait mean decline from $3.88 \pm 0.06$ to $3.74 \pm 0.10$. Estimates of inbreeding depression from purebred groups were larger in magnitude than for crossbred groups but the effect was not significant (difference $0.57 \pm 0.42$ ). While effects of the year of birth (estimates not shown) were significant, there was no evidence of a consistent long-term trend. In combined group analyses, separate levels of fixed effects (including inbreeding depression) were estimated for each group.

Table II. $N_{s} / N_{p}$ separate group results.

\begin{tabular}{lcccccccccccc}
\hline Breed & \multicolumn{2}{c}{$V_{A}$} & \multicolumn{2}{c}{$V_{e}$} & \multicolumn{2}{c}{$V_{d r}$} & \multicolumn{2}{c}{ I.D. } & Mean & $l_{1}$ \\
\hline Bf & $0.16^{*}$ & $(0.11)$ & 0.09 & $(0.09)$ & 0.24 & $(0.18)$ & -1.46 & $(0.64)$ & 3.33 & $(0.24)$ & -14.4 \\
Ch & $0.20^{*}$ & $(0.15)$ & 0.24 & $(0.15)$ & 0.16 & $(0.28)$ & -0.32 & $(0.76)$ & 4.16 & $(0.21)$ & -34.5 \\
We & \multicolumn{3}{c}{0.0} & 0.23 & $(0.07)$ & $0.16^{*}$ & $(0.10)$ & -0.80 & $(0.48)$ & 3.61 & $(0.23)$ & -9.3 \\
Bf-Ch & $0.15^{*}$ & $(0.09)$ & 0.14 & $(0.08)$ & $0.35^{*}$ & $(0.16)$ & -0.17 & $(0.45)$ & 3.6 & $(0.17)$ & -67.8 \\
Bf-We & $0.28^{* *}$ & $(0.09)$ & 0.14 & $(0.06)$ & 0.12 & $(0.12)$ & -0.53 & $(0.40)$ & 3.95 & $(0.15)$ & -38.9 \\
Ch-We & 0.10 & $(0.07)$ & 0.12 & $(0.07)$ & $0.31^{*}$ & $(0.14)$ & -0.23 & $(0.45)$ & 4.16 & $(0.19)$ & -31.1 \\
\hline
\end{tabular}

Variance components, inbreeding depression (I.D.), trait means and log likelihood $\left(l_{1}\right)$ for separate group analyses. Standard errors follow estimates except where the component has been constrained to zero to satisfy feasibility. Breed groups are abbreviated as follows: Bf: Scottish Blackface; Ch: South Country Cheviot; We: Welsh Mountain. Results of likelihood ratio tests for significance are indicated by asterisks: ${ }^{*} P<0.05,{ }^{* *} P<0.01$.

\subsection{Variance components for $N_{s} / N_{p}$}

Variance components results in table $I I$ are from a reduced model including only $V_{A}, V_{e}$ and $V_{d r}$. In most cases it was not possible to estimate variances due to homozygous dominance, i.e. $D_{1}, D_{2}^{*}$ and $H^{*}$. These were difficult to estimate because large negative sampling correlations between $D_{2}^{*}$ and both $V_{A}$ and $V_{e}$ [5] resulted in infeasible estimates at best, and instability of the Fisher scoring maximization algorithm at worst.

\subsubsection{Additive variance}

Additive variance was detected $(P<0.05)$ in all of the groups except for Welsh and Cheviot-Welsh (table $I I)$. Heritability estimates ranged from 0 to 0.51 .

There was no evidence from the likelihood tests for differences in the additive component within the purebred and crossbred groups nor between purebred $\left(h^{2}=0.31\right)$ and crossbred $\left(h^{2}=0.35\right)$ combined data sets (table III).

\subsubsection{Dominance variance}

In Welsh, Blackface-Cheviot and Cheviot-Welsh breeds, there was evidence of random dominance variance $(P<0.05)($ table $I I)$. In the pooled data 
(table III), there was evidence of random dominance in the crossbred groups but not in the purebred groups, although the difference in magnitude between them was not significant. Homozygous dominance variance components either could not be estimated, or did not differ significantly from zero for these data sets.

Table III. $N_{s} / N_{p}$ combined group results.

\begin{tabular}{|c|c|c|c|c|c|c|c|c|c|}
\hline \multicolumn{2}{|c|}{$V_{A}$} & & $V_{e}$ & & $V_{d r}$ & $D_{1}$ & & $D_{2}^{*}$ & $l_{1}$ \\
\hline \multicolumn{10}{|c|}{ Combined purebred groups } \\
\hline $0.15^{*}$ & $(0.06)$ & 0.20 & $(0.06)$ & 0.13 & $(0.11)$ & 0.0 & 0.0 & $(0.0)$ & -63.58 \\
\hline \multicolumn{10}{|c|}{ Combined crossbred groups } \\
\hline $0.20^{* *}$ & $(0.05)$ & 0.14 & $(0.04)$ & $0.23^{*}$ & ** $(0.08)$ & & & & -142.1 \\
\hline 0.17 & $(0.07)$ & 0.11 & $(0.07)$ & 0.28 & $(0.12)$ & 0.0 & 0.10 & $(0.19)$ & -142.0 \\
\hline
\end{tabular}

Variance components and log likelihood $\left(l_{1}\right)$ for combined group analyses. In the case of the crossbred groups, results are included for analyses with and without the components $D_{1}$ and $D_{2}^{*}$. Standard errors follow estimates in parentheses except where the component has been constrained to zero to satisfy feasibility. Results of likelihood ratio tests for significance are indicated by asterisks: ${ }^{*} P<0.05,{ }^{* *} P<0.01$.

When the two forms of dominance variance $V_{d r}$ and $D_{2}^{*}$ were combined in the pooled dominance model, the resulting dominance component was again significant for the crossbreds but not for the purebreds. The pooled dominance model resulted in a higher likelihood in the purebreds over the model with $D_{2}^{*}=D_{1}=0$, but no change in likelihood in the crossbreds. Since these models are not nested, a test was not possible.

\subsection{Fixed effects for weight}

The fixed effects significantly affecting the weight measurement data were, for dams, inbreeding coefficient, age and parity, and for lambs, year of birth, inbreeding coefficient and birth/rearing type (born single/reared single, born as twin/reared as twin, etc.). The influences of the dam's attributes and the birth/rearing type were much less in the 78-week weight than in the 24-week weight.

Inbreeding depression for the lamb (table IV) was observed within all breed types and was much greater for the 78 -week weight than for the 24 -week weight. As in the $N_{s} / N_{p}$ trait, inbreeding depression for weight in the crossbred groups tended to be lower in magnitude than expected if the inbreeding depressions of the constituent purebred groups were averaged; but again this was not significant. The trait mean for each crossbred was very close to the average of the means of the constituent purebred groups for both 24-week weight and 78-week weight. 


\subsection{Variance components for weight}

\subsubsection{Additive variance}

A substantial amount of additive variation was evident in all groups at both ages (table $I V$ ). Heritabilities ranged from 0.20 to 0.46 for 24 -week weight and from 0.12 to 0.69 for 78 -week weight.

Table IV. Weight results from separate group analyses.

\begin{tabular}{|c|c|c|c|c|c|c|c|c|}
\hline Breed & & $V_{A}$ & & $V_{e}$ & $V_{d r}$ & & $V_{m}$ & I.D. \\
\hline \multicolumn{9}{|c|}{$\begin{array}{l}\text { Purebred groups } \\
24 \text {-week weights }\end{array}$} \\
\hline $\mathrm{Bf}$ & 11.5 & $(4.4)^{* *}$ & 9.4 & $(3.5)$ & $0.4 \quad(5.0)$ & 3.6 & $(1.9)^{*}$ & $-13.8(2.1)$ \\
\hline $\mathrm{Ch}$ & 7.4 & $(5.1)^{* *}$ & 15.7 & $(6.3)$ & $0.5 \quad(9.5)$ & & 0.0 & $-14.4(4.0)$ \\
\hline We & 3.7 & $(1.5)^{* *}$ & 3.4 & $(0.9)$ & 0.0 & 3.4 & $(0.9)^{* *}$ & $-10.5(1.3)$ \\
\hline \multicolumn{9}{|c|}{ 78-week weights } \\
\hline $\mathrm{Bf}$ & 30.1 & $(8.3)^{* *}$ & 8.3 & $(5.2)$ & $8.9 \quad(7.7)$ & & 0.0 & $-25.1(2.4)$ \\
\hline $\mathrm{Ch}$ & 6.2 & $(8.8)^{* *}$ & 14.4 & $(11.0)$ & $29.3(20.2)$ & 0.0 & $(5.4)$ & $-29.9(5.5)$ \\
\hline $\mathrm{We}$ & 14.1 & $(3.6)^{* *}$ & 5.84 & $(1.9)$ & 0.0 & 1.7 & $(1.3)$ & $-17.4(1.7)$ \\
\hline \multicolumn{9}{|c|}{ Crossbred groups } \\
\hline \multicolumn{9}{|c|}{ 24-week weights } \\
\hline $\mathrm{Bf}-\mathrm{Ch}$ & 5.6 & $(2.4)^{* *}$ & 5.2 & $(2.2)$ & $8.7(3.7)^{*}$ & 7.2 & $(1.6)^{* *}$ & $-10.5(1.5)$ \\
\hline Bf-We & 7.9 & $(2.1)^{* *}$ & 6.0 & $(1.8)$ & $1.5(2.6)$ & 3.7 & $(1.2)^{* *}$ & $-12.5(1.3)$ \\
\hline Ch-We & 6.6 & $(2.2)^{* *}$ & 4.0 & $(1.9)$ & $4.8(2.8)^{*}$ & 1.8 & $(1.1)$ & $-11.9(1.4)$ \\
\hline \multicolumn{9}{|c|}{ 78-week weights } \\
\hline $\mathrm{Bf}-\mathrm{Ch}$ & 23.9 & $(5.3)^{* *}$ & 10.5 & $(3.5)$ & $5.8(4.7)$ & 2.8 & $(2.0)^{*}$ & $-25.4(1.7)$ \\
\hline Bf-We & 20.4 & $(4.5)^{* *}$ & 7.7 & $(3.1)$ & $4.4(4.6)$ & 4.5 & $(2.0)^{*}$ & $-21.7(1.7)$ \\
\hline $\mathrm{Ch}-\mathrm{We}$ & 21.8 & $(4.4)^{* *}$ & 9.9 & $(2.1)$ & 0.0 & & 0.0 & $-21.7(1.7)$ \\
\hline
\end{tabular}

Variance components and inbreeding depression (I.D.) for separate group analyses of 24-week and 78-week live weight. Standard errors follow estimates except where the component has been constrained to zero to satisfy feasibility. Breed groups are abbreviated as follows: Bf: Scottish Blackface; Ch: South Country Cheviot; We: Welsh Mountain. Results of likelihood ratio tests for significance are indicated by asterisks: ${ }^{*} P<0.05,{ }^{* *} P<0.01$.

\subsubsection{Dominance and maternal variance}

Much of the information in the data used to calculate random dominance variance comes from full-sib groups and thus we find substantial sampling correlations between estimates for $V_{d r}$ and $V_{m}$. Significant $V_{d r}$ could not be detected in any of the purebred groups for 24-week weight though it is present $(P<0.05)$ in two of the three crossbred groups. For 78 -week weight there was no evidence of random dominance in any of the separate group data sets (see (table $I V)$. 
Maternal variance in the separate group analyses (table IV) was generally higher for 24-week weight than it was for 78-week weight, confirming the observation of reduced influence of the dam from the fixed effects. Among the purebred groups, significant maternal variance was found in all but the Cheviot group for 24-week weight but could not be detected for 78 -week weight. The crossbred groups, with the exception of the Cheviot-Welsh breed, showed significant maternal variance for both 24- and 78-week weight.

Sample size and pedigree structure were never adequate to estimate components of variance involving homozygous dominance as distinguished from random dominance within each group. When the pooled dominance model was fitted, the combined dominance component was present in the Blackface and Blackface-Cheviot groups for both 24- and 78-week weight (results not shown). Correlations between homozygous dominance and additive effects $\left(D_{1}\right)$ were negative for both the Blackface and the Blackface-Cheviot breeds but was only significantly different from zero $(P<0.05)$ in the Blackface breed.

The results of the analysis of the combined purebreds and combined crossbreds are complicated since there was evidence of differences in the components, among the combined groups, that were statistically significant and not strictly related to the mean weight. Therefore, the constraint that the three groups in each combined data set share a single set of variance components may have introduced bias into the combined results and table $V$ must be viewed in this context. The only significant homozygous dominance variance in these combined analyses was in the combined purebred data set. The magnitude of this variance was not significantly different between 24 and 78 weeks although the higher additive variance at 78 weeks implies a smaller negative correlation between additive and homozygous dominance effects at the later age $(-0.91$ at 24 weeks, -0.80 at 78 weeks).

The combined crossbred line data sets showed very little evidence of homozygous dominance variance. In the case of the 78-week weights, where the combining of the constituent crossbred data sets required the least constraint, the inclusion of homozygous dominance variance components in the model increased the log likelihood by a mere 0.22 . Although the estimates for the two dominance variance components in the combined crossbred data set are very nearly the same, the constraint that these two were the same (pooled dominance model) results in a slightly poorer fit than that given by the model in which the homozygous dominance components are constrained to zero. Since these models are not nested, a test was not possible. This indicates, however, that our power to detect homozygous dominance variance components is inadequate even though random dominance can be quite accurately estimated. Homozygous dominance variance is also not to be found in the 24-week data which, nevertheless, show highly significant random dominance variance. The pooled dominance model again in this case results in a negligible likelihood difference.

\section{DISCUSSION}

The structure of the data analyzed, and their size, are unique in livestock and unusual in mammals, with rapid inbreeding conducted up to and beyond $50 \%$ in a variety of pure breeds, and crosses of those breeds, followed by crossing 
Table V. Combined line weight results.

\begin{tabular}{|c|c|c|c|c|c|c|c|}
\hline & $V_{a}$ & $V_{e}$ & $V_{d r}$ & $V_{m}$ & $D_{1}$ & $D_{2}^{*}$ & $l_{1}$ \\
\hline \multicolumn{8}{|c|}{$\begin{array}{l}\text { Purebred groups } \\
24 \text {-week weights }\end{array}$} \\
\hline 7.9 & $(1.8)^{* *}$ & $7.3(1.1)$ & 0.0 & $2.7(0.9)^{* *}$ & & & -1278.7 \\
\hline 16.7 & $(3.2)^{* *}$ & $8.1(1.8)$ & 0.0 & $2.5(0.8)^{* *}$ & $-14.6(4.6)^{* *}$ & $30.3(12.5)$ & -1272.8 \\
\hline \multicolumn{8}{|c|}{ 78-week weights } \\
\hline 17.2 & $(3.6)^{* *}$ & $10.9(2.8)$ & $3.8(4.2)$ & 1.1 (1.3) & & & -1456.4 \\
\hline 29.9 & $(6.0)^{* *}$ & $12.0(3.2)$ & 0.0 & $1.2(1.3)$ & $-16.3(7.9)^{*}$ & $27.5(20.2)$ & -1453.9 \\
\hline \multicolumn{8}{|c|}{$\begin{array}{l}\text { Crossbred groups } \\
24 \text {-week weights }\end{array}$} \\
\hline 6.1 & $(1.3)^{* *}$ & $5.6(1.1)$ & $5.8(1.9)^{* *}$ & $3.7(0.7)^{* *}$ & & & -2789.0 \\
\hline 6.8 & $(2.1)^{* *}$ & $6.9(1.2)$ & $4.4(2.0)^{* *}$ & $3.9(0.7)^{* *}$ & $0.34 \quad(2.3)$ & $-6.5(5.9)$ & -2788.0 \\
\hline \multicolumn{8}{|c|}{ 78-week weights } \\
\hline 19.4 & $(2.6)^{* *}$ & $9.1(1.7)$ & $5.0(2.6)^{*}$ & $2.5(0.9)^{* *}$ & & & -3108.7 \\
\hline 20.2 & $(3.8)^{* *}$ & $8.5(2.1)$ & $5.2(3.1)^{*}$ & $2.2(0.9)^{* *}$ & $-1.9 \quad(4.3)$ & $6.6(10.9)$ & -3108.5 \\
\hline
\end{tabular}

Variance components and $\log$ likelihood $\left(l_{1}\right)$ for combined group analyses of 24 -week and 78-week live weight. Two models are shown for each data set, one with and one without the homozygous dominance variance components $D_{1}$ and $D_{2}^{*}$. Standard errors follow estimates in parentheses except where the component has been constrained to zero to satisfy feasibility. Results of likelihood ratio tests for significance are indicated by asterisks: ${ }^{*} P<0.05,{ }^{* *} P<0.01$.

of the inbred lines within a breed type. Despite this structure the estimation of the variance components pertaining to homozygous dominance effects was found to be difficult. For the ratio of secondary to primary follicle numbers, random dominance variance was estimated to be of a similar magnitude to additive and to environmental variance, depression to complete inbreeding was of the order of 1 environmental standard deviation, but parameters pertaining to inbred dominance effects were negligible. For live weight, random dominance variance was usually smaller in magnitude than additive variance and depression to complete inbreeding was five or six times the environmental standard deviation, with estimates of inbred dominance variance components rarely obtainable and even more rarely significant. At all times $H^{*}$, the sum of the squared homozygous deviations, was small or was estimated to be negative and consequently constrained to zero. The interpretation of such results is difficult.

For live weight, it is easiest to postulate many loci, each having alleles of small effect: thus, the total sum of the homozygous dominance effects may be substantial (hence, a large inbreeding depression) but $H^{*}$ is small. If a biallelic model is considered at each locus, then a model of rare recessives of large effect could be considered to fit the pattern observed. The inbreeding depression per locus would be approximately $p_{r} d_{r r}$ (where $r$ denotes the recessive allele) for each locus and could be of significant magnitude when summed over loci if each term were $O(1 / N)$ where $N$ is the number of loci. $H^{*}$ would be approximately $\left(p_{r} d_{r r}\right)^{2}$ and consequently very much smaller when $N$ is large. $D_{2}^{*}$ would be 
approximately $4 p_{r}\left(d_{r r}\right)^{2}$, which is not necessarily small. For the follicle ratio this explanation of many loci with rare recessives is less satisfactory since the inbreeding depression is weak while $V_{d r}$ is large.

This tentative conclusion for live weight appears to agree with those of Caballero and Keightley [1] for the quantitative trait of bristle number in Drosophila melanogaster. From an analysis of the distributions of $P$-element mutagenesis, they concluded that a large proportion of the additive variance and practically all of the dominance variance arose from recessive or partially recessive mutants. The relative magnitude of the partition between the additive and dominance variance found in their study was 8 to 1 , which has some similarity to the relative magnitude observed here. Davis et al. [6] observed that a few genes of large effect may explain some $75 \%$ of the additive genetic variation for birth weight in a crossbred population. Such genes are predicted to be at least partially recessive in the distributions of Caballero and Keightley [1]. There was no indication in the analysis by Davis et al. [6] of whether dominance was found with these loci, although the experimental design should give some information on this.

Another complication requiring consideration is the sensitivity of dominance components to epistasis. Epistasis has been observed for loci of large effect on bristle number [13] and the presence of epistasis in previous analyses of this data set were significant for live weight [25] but not for $N_{s} / N_{p}$ [30]. Such a sensitivity to at least some form of epistasis (which is a very general term covering all interactions among loci) might be expected. The possibility of errors in estimation can be discounted since all the estimates from the simulation study were consistent with the true parameters in the absence of selection.

Recent treatments in the animal breeding literature of the problem of dominance variance with inbreeding have proposed that the quadratic components of variance which involve homozygous dominance variance might safely be ignored when genetic evaluation is the goal of the analysis [7, 21]. The current analysis adds credence to this contention. Not only are the homozygous dominance components very difficult to calculate, but they appear to be absent or indistinguishable from random dominance in the largest and most thorough analyses yet completed despite the presence of substantial random dominance and inbreeding depression. However, the development of finite locus models [17] would prevent the need for this choice between incompleteness and the complexity of the components.

\section{ACKNOWLEDGEMENTS}

J.A.W. gratefully acknowledges funding by the Ministry of Agriculture, Fisheries and Food (UK). F.H.S. gratefully acknowledges support from Pioneer Hibred International, Inc., to develop the computer programs used in this paper, and a grant from the Underwood fund of the Biotechology and Biological Sciences Research Council (UK) to support a prolonged sojourn at the Roslin Institute, Edinburgh.

\section{REFERENCES}

[1] Caballero A., Keightley P.D., A pleiotropic nonadditive model of variation in quantitative traits, Genetics 138 (1994) 883-900. 
[2] Carter H.B., Clarke W.H., The hair follicle group and skin follicle population of Australian merino sheep, Austr. J. Agric. Res. 8 (1957) 91-108.

[3] Cockerham C.C., Higher order probability functions of identity of alleles by descent, Genetics 69 (1971) 235-246.

[4] Cockerham C.C., Weir, B.S., Covariances of relatives stemming from a population undergoing mixed self and random mating, Biometrics 40 (1984) 157-164.

[5] Cornelius P.L., Properties of components of covariance of inbred relatives and their estimates in a maize population, Theor. Appl. Genet. 75 (1988) 701-711.

[6] Davis G.P., Hetzel D.J.S., Corbet N.J., Scacheri S., Lowden S., Renaud J., Mayne C., Stevenson R., Moore S.S., Byrne K., The mapping of quantitative trait loci for birth weight in a tropical beef herd, in: Proceedings of the 6th World Congress on Genetics Applied to Livestock Production, vol. 26, 1998, pp. 441-444.

[7] de Boer I. J.M., Hoeschele, I., Genetic evaluation methods for populations with dominance and inbreeding, Theor. Appl. Genet. 86 (1993) 245-258. 1989.

[8] Falconer D.S., Introduction to Quantitative Genetics, Longman Group, London,

[9] Harris D.L., Genotypic covariances between inbred relatives, Genetics 50 (1964) 1319-1348.

[10] Hill W.G., Robertson R., The effects of inbreeding at loci with heterozygote advantage, Genetics 60 (1968) 615-628.

[11] Lo L.L., Fernando R.L., Cantet R.J.C, Grossman M., Theory of modelling means and covariances in a two-breed population with dominance, Theor. Appl. Genet. 90 (1995) 49-62.

[12] Lo L.L., Fernando R.L., Grossman M., Genetic evaluation by BLUP in twobreed terminal crossbreeding systems with dominance, J. Anim. Sci. 75 (1997) 28772884 .

[13] Mackay T.F.C., The nature of quantitative variation: lessons from Drosophila, in: Proceedings of the 6th World Congress on Genetics Applied to Livestock Production, vol. 26, 1998, pp. 69-76.

[14] Meyer K., Maximum likelihood procedures for estimating genetic parameters for later lactations of dairy cattle, J. Dairy Sci. 66 (1983) 1988-1997.

[15] Meyer K., Smith S.P., Maximum likelihood estimation for animal models using derivatives of the likelihood, Genet. Sel. Evol. 28 (1996) 23-49.

[16] Meyer K., An 'average information' restricted maximum likelihood algorithm for estimating reduced rank genetic covariance matrices or covariance functions for animal models with equal design matrices, Genet. Sel. Evol. 29 (1997) 97-116.

[17] Pong-Wong P., Shaw F.H., Woolliams J.A., Estimation of dominance variation using a finite-locus model, in: Proceedings of the 6th World Congress on Genetics Applied to Livestock Production, vol. 26, 1998, pp. 41-44.

[18] Searle S.R., Casella G., McCulloch C.E., Variance Components, John Wiley, New York, 1993.

[19] Self S.G., Liang K., Asymptotic properties of maximum likelihood estimators and likelihood ratio tests under nonstandard conditions, J. Am. Stat. Assoc. 82 (1990) 605-610.

[20] Smith S.P., Maki-Tanila A., Genetic covariance matrices and their inverses for models allowing dominance and inbreeding, Genet. Sel. Evol. 22 (1990) 65-91.

[21] Uimari P., Kennedy B.W., Mixed model methodology to estimate additive and dominance genetic values under complete dominance, in: Hill W.G., Thompson R., Woolliams J.A. (Eds.), Proceedings of the 4th World Congress of Genetics Applied to Livestock Production, vol. 13, Edinburgh, Edinburgh University Press, 1990, pp. 297300 .

[22] Wei, M. van der Werf J.H.J., Maximizing genetic response in crossbreds using both purebred and crossbred information, Anim. Prod. 58 (1994) 401-413. 
[23] Weir B. S. Cockerham, C.C., Two locus theory in quantitative genetics, in: Pollak E., Kempthorn O., Bailey T.B.J. (Eds.), Proceedings of the International Conference on Quantitative Genetics, Iowa State University Press, Ames, IA, 1977, pp. 247-269.

[24] Wiener G., A comparison of the body size, fleece weight and maternal performance of five breeds of sheep kept in one environment, Anim. Prod. 9 (1967) $177-195$.

[25] Wiener G., Lee G.J., Woolliams J.A., Effects of rapid inbreeding and of crossing inbred lines on the body weight growth of sheep, Anim. Prod. 55 (1992) 89-99.

[26] Wiener G., Lee G.J., Woolliams J.A., Effects of rapid inbreeding and of crossing inbred lines on the growth of linear body dimensions of sheep, Anim. Prod. 55 (1992) 101-114.

[27] Wiener G., Lee G.J., Woolliams J. A., Effects of rapid inbreeding and of crossing inbred lines on the conception rate, prolificacy and ewe survival in sheep, Anim. Prod. 55 (1992) 115-121.

[28] Wiener G., Lee G.J., Woolliams J.A., Consequences of inbreeding for financial returns from sheep, Anim. Prod. 59 (1994) 245-249.

[29] Woolliams J.A., Wiener G., The effects of breed, crossbreeding and other factors on variation in fleece and skin traits, Anim. Prod. 30 (1980) 417-429.

[30] Woolliams J.A., Wiener, G., The effect of breed type and inbreeding on characteristics of the fleece and skin of 12-week-old lambs, Anim. Prod. 32 (1981) 9-22.

[31] Wright S., Systems of mating, Genetics 6 (1921) 111-178. 